\title{
Acute Generalized Exanthematous Pustulosis
}

National Cancer Institute

\section{Source}

National Cancer Institute. Acute Generalized Exanthematous Pustulosis. NCI Thesaurus. Code C112122.

A widespread acute rash characterized by fever and multiple small pustules on a reddish background. 